\section{Transtornos mentais comuns e auto-estima na gestação: prevalência e fatores associados}

\author{
Common mental disorders and self-esteem in \\ pregnancy: prevalence and associated factors
}

\author{
${ }^{1}$ Universidade Católica de \\ Pelotas, Pelotas, Brasil. \\ Correspondência \\ L. C. Ores \\ Universidade Católica de \\ Pelotas. \\ Av. Almirante Barroso 1202 \\ sala 109G, Pelotas, RS \\ 96010-280, Brasil. \\ lilianeores@hotmail.com
}

\begin{abstract}
The aim of this study was to assess the prevalence of common mental disorders and the association with self-esteem and other factors in pregnant women. A nested cross-sectional study was performed in a cohort of pregnant women treated in the public health system in Pelotas, Rio Grande do Sul State, Brazil. The Self-Reporting Questionnaire (SRQ-20) was used to screen for common mental disorders and the Rosenberg's Self-Esteem Scale for self-esteem. The sample consisted of 1,267 pregnant women with a mean age of 25 years ( $S D=6.53)$. Mean self-esteem was 9.3 points $(S D=4.76)$, and prevalence of common mental disorders was $41.4 \%$. Lower self-esteem was associated with higher odds of common mental disorders $(p<0.001)$. There was a significant association between higher prevalence of common mental disorders and low self-esteem.
\end{abstract}

Pregnancy; Self Esteem; Mental Disorders
Ricardo Azevedo da Silva 1

Liliane da Costa Ores 1

Thaíse Campos Mondin 1

Raquel Nolasco Rizzo

Inácia Gomes da Silva Moraes 1

Karen Jansen 1

Ricardo Tavares Pinheiro ${ }^{1}$

\section{Introdução}

A gestação é um período de transição que faz parte do desenvolvimento humano. Há transformações no organismo da mulher e no seu bemestar, alterando seu psiquismo e o seu papel sociofamiliar, podendo assim, ser um período em que se observam aumentos de sintomatologias, ou mesmo, o desenvolvimento de transtornos psiquiátricos 1,2 .

Transtornos mentais comuns (TMC) incluem sintomas depressivos não-psicóticos, ansiedade e queixas somáticas que afetam o desempenho das atividades diárias. Os sintomas dessa categoria são: dificuldade de concentração e de esquecimento, insônia, fadiga, irritabilidade, sensação de inutilidade, e queixas somáticas, entre outros ${ }^{3}$. Na população geral, os TMC apresentam prevalência de $22,7 \%$ (17,9\% entre os homens e $26,5 \%$ entre as mulheres) ${ }^{4}$. Enquanto estudos com a população de gestantes e mesmo instrumento de aferição têm encontrado prevalência um pouco maior, sendo $33,6 \%$ em estudo no Paraguai 5 e 43,1\% em Recife no Brasil 6 .

Pesquisas têm mostrado vários fatores associados aos TMC, tais como, baixo nível de escolaridade e/ou socioeconômico, maior faixa etária, sexo feminino, ser solteiro ou estar divorciado, estar desempregado ou não ter nenhuma ocupação, tabagismo, alcoolismo, sedentarismo e insatisfação com a imagem corporal $4,5,6,7,8,9,10,11,12,13$. 
No Brasil, a temática da auto-estima é pouco abordada cientificamente 14 . Níveis elevados de auto-estima têm sido apontados por estudos como importante preditor de competências maternas e de alta qualidade na interação mãe/bebê 15 . A auto-estima pessoal diz respeito à avaliação positiva ou negativa que o indivíduo faz de si mesmo, constituindo-se, assim, em um aspecto central do eu, que desempenha papel fundamental no processo de construção da identidade adulta. Ela é, portanto, uma conseqüência da identidade pessoal, congregando, desta forma, as auto-avaliações sobre os atributos e habilidades individuais manifestos nos domínios interpessoal e privado 16 .

Embora os temas TMC e auto-estima na gestação sejam relevantes para um maior entendimento do impacto dos transtornos mentais sobre as gestantes, não foram encontrados estudos sobre esta associação.

O objetivo deste estudo foi verificar a prevalência de transtornos mentais comuns e sua relação com auto-estima, bem como outros fatores associados à ocorrência de TMC em gestantes. Tendo como hipótese que as mulheres com menor auto-estima têm maior probabilidade de apresentar TMC.

\section{Métodos}

Este é um estudo transversal que faz parte de uma coorte perinatal para avaliação de transtornos mentais, tendo como população-alvo gestantes assistidas pelo Programa de Humanização no Pré-Natal e Nascimento (PHPN), do Sistema Único de Saúde (SUS) da cidade de Pelotas, Rio Grande do Sul. Assim, foram captadas as gestantes atendidas pelas 38 Unidades Básicas de Saúde (UBS) cadastradas no Sistema de Informação em Saúde Pré-natal (SisPreNatal) do Departamento de Informática do SUS (DATASUS). Foram excluídas as gestantes que não vivem na zona urbana de Pelotas, ou ainda, que mostraram incapacidade de compreender ou responder ao questionário. A inclusão foi independente da faixa etária e período gestacional, ou seja, todas a mulheres cadastradas no SisPreNatal eram elegíveis para participar do estudo.

A captação da amostra foi realizada diariamente, entre 2006 e 2008, por estudantes do curso de psicologia da Universidade Católica de Pelotas (UCPel), a fim de explicar o inquérito e obter o consentimento livre e esclarecido para posterior aplicação dos instrumentos no domicílio das gestantes. Foi utilizado um questionário para as informações pré-natais, padronizado com questões sobre a idade materna, escolarida- de, estado civil, planejamento da gravidez, pensamentos em aborto, entre outros fatores.

No intuito de verificar a situação socioeconômica, foi utilizado o instrumento da ABEP (Associação Brasileira de Empresas de Pesquisa). Essa classificação seleciona os indivíduos por índice de conforto, tais como: televisão, geladeira, rádio, automóvel e empregadas domésticas, além da escolaridade da pessoa de maior renda na casa, classificando em A, B, C, D e E, em que "A" referese à classificação socioeconômica mais alta e "E" à mais baixa.

Foi utilizado para o rastreamento de TMC o Self-Reporting Questionnaire (SRQ-20), indicado pela Organização Mundial da Saúde (OMS) para estudos comunitários e em atenção básica à saúde. O instrumento é composto por 20 questões para o rastreamento de transtornos não-psicóticos e as respostas são dicotômicas, sendo que, cada afirmativa soma um ponto, em um escore de 0 a 20 que varia de nenhuma à extrema probabilidade, respectivamente. O SRQ-20 é um instrumento de rápida e fácil aplicação, de baixo custo e não requer a presença de um entrevistador clínico. O ponto de corte utilizado para discriminar as gestantes com TMC foi 7 , que de acordo com a validação brasileira apresenta os seguintes padrões psicométricos: sensibilidade de $86,33 \%$ e especificidade de $89,31 \% 17$.

Para avaliar a auto-estima foi utilizada a $E s$ cala de Auto-Estima de Rosenberg, validada por Dini 18. Esse instrumento é composto por dez afirmativas, cada uma com quatro opções de respostas que variam de zero a três, ou seja, de nada importante à extremamente importante. Desse modo, o escore final da escala pode variar de zero (melhor auto-estima) a trinta (pior auto-estima).

Para detecção de transtornos por uso de álcool foi utilizado o questionário CAGE, validado no Brasil em 1983 por Masur \& Monteiro 19, que encontraram uma sensibilidade de $84,7 \%$ e uma especificidade de $73,3 \%$ para o ponto de corte maior ou igual a dois.

Gestantes que apresentaram TMC ou menor auto-estima mereceram especial atenção, sendo encaminhadas para tratamento no Ambulatório de Psiquiatria do Campus da Saúde da UCPel. Este projeto de pesquisa foi aprovado pelos Comitês de Ética em Pesquisa da Universidade Católica de Pelotas e da Universidade Federal de Pelotas, sob o protocolo de número 2006/49, e autorizado pela Secretaria de Saúde da Prefeitura Municipal de Pelotas.

O Epi Info (Centers for Disease Control and Prevention, Atlanta, Estados Unidos) foi usado para digitação dos dados e checagem automática para a amplitude e consistência. As análises estatísticas foram realizadas pelo programa Stata 
(Stata Corp., College Station, Estados Unidos). Após a obtenção da freqüência simples de todas as variáveis, foi feita a análise bruta usando-se o teste qui-quadrado para a associação entre as variáveis independes e TMC, enquanto a análise da associação entre auto-estima e TMC foi realizada pelo teste $t$ de Student para comparação entre as médias. Todas as variáveis em estudo que obtiveram $\mathrm{p}<0,20$ na análise bruta foram incluídas na análise ajustada.

Na análise ajustada, utilizou-se a regressão de Poisson para avaliar a razão de prevalência entre as variáveis independentes estudadas e a presença de TMC. Nesse tipo de análise, o modelo hierárquico constituiu-se de variáveis demográficas e socioeconômicas no primeiro nível - faixa etária, escolaridade, classificação socioeconômica, trabalho e estado civil; variáveis psicossociais no segundo nível - apoio familiar, planejamento da gestação e pensamento sobre aborto; e, consumo de substâncias psicoativas lícitas nos últimos três meses prévios à entrevista no terceiro nível - consumo de tabaco e bebida alcoólica. Conceitualmente, considera-se assim que as variáveis demográficas e socioeconômicas têm relação entre si; estas influenciam o círculo social das pessoas e os aspectos psicológicos na tomada de decisões, e, por fim, exercem influência sobre o consumo de substâncias, que embora se relacionem, não há certeza sobre a temporalidade destes eventos 20 .

Após análise ajustada foram consideradas associações significativas quando $\mathrm{p}<0,05$.

\section{Resultados}

O total de 1.340 gestantes foram identificadas, sendo que $6 \%$ recusaram-se a participar do estudo ou foram excluídas, assim, a amostra constitui-se de 1.267 gestantes. Essas tinham em média 25 anos ( $d p=6,53)$, sendo que $43 \%$ eram analfabetas ou não completaram o Ensino Fundamental, 53,6\% pertenciam à classe "C" (média), $69,6 \%$ não estavam trabalhando no momento da entrevista e $73,2 \%$ eram casadas ou viviam com o companheiro. Além disso, 95,6\% das mulheres sentiam-se apoiadas pela família, $41,9 \%$ não planejaram a gestação, e 9,4\% pensaram em aborto. Em se tratando do uso de substâncias psicoativas, $19,3 \%$ das grávidas consumiam tabaco e $8,2 \%$, bebida alcoólica (Tabela 1 ).

Considerando que o escore pode variar de zero (maior auto-estima) a trinta (menor autoestima), as gestantes apresentaram em média uma auto-estima de 9,3 pontos $( \pm 4,76)$. Maiores escores (menor auto-estima) foram encontrados entre as gestantes que estavam no segundo tri- mestre gestacional ( $p=0,015)$, nas mais jovens ( $\mathrm{p}=0,014)$, nas de menor escolaridade $(\mathrm{p}<$ 0,001 ), aquelas que não viviam com o companheiro ( $p=0,006$ ), as que tinham menor classificação socioeconômica ( $p<0,001$ ), as que não trabalhavam $(\mathrm{p}<0,001)$, aquelas que não tinham suporte familiar $(p=0,016)$, nas que pensaram em abortar $(p<0,001)$ e nas que consumiram álcool ( $\mathrm{p}<0,001)$ ou que fumaram durante a gestação $(\mathrm{p}<0,001)$.

A prevalência de transtornos mentais comuns entre as gestantes avaliadas foi de $41,4 \%$. A Tabela 1 também descreve as características das gestantes com presença de TMC, as quais, $41,8 \%$ tinham de 19 a 34 anos de idade, $47 \%$ eram analfabetas ou não concluíram o Ensino Fundamental, 48,8\% situavam-se nas classes " $D$ " ou "E" de acordo com a ABEP, 43,9\% não estavam trabalhando, 44,3\% eram solteiras, divorciadas ou viúvas, $42,4 \%$ estavam no segundo trimestre gestacional, 65,5\% não se sentiam apoiadas pela família, 34,2\% não planejaram a gestação, 70,3\% pensaram em abortar o bebê, $53,1 \%$ consumiam tabaco e 58,6\% consumiam bebida alcoólica.

$\mathrm{Na}$ análise bruta, a presença de TMC esteve associada de forma significativa à menor escolaridade, à classificação socioeconômica inferior, não estar trabalhando, não se sentir apoiada pela família, ter pensado em abortar, ter consumido álcool e bebida alcoólica durante a gestação (Tabela 2). Além disso, relato de menor autoestima da grávida esteve associado à ocorrência de TMC.

Na Tabela 2, ressalta-se ainda que, na análise ajustada, a presença de TMC apresentou-se significativamente associada à menor escolaridade, classificação socioeconômica inferior, não se sentir apoiada pela família e ter pensado em abortar. Evidencia-se, também, que quanto menor a auto-estima da grávida maior a probabilidade dela apresentar TMC.

\section{Discussão}

O principal achado deste estudo transversal com gestantes atendidas pelo serviço público na cidade de Pelotas foi a alta prevalência de TMC nessa amostra e sua significativa associação com menor auto-estima.

Neste estudo, foi encontrada uma prevalência de TMC em gestantes de $41,4 \%$, alta quando comparada a estudos populacionais que evidenciam prevalências em torno de $17 \%$ a $27 \%$ $4,5,11,21,22$, mas semelhante à encontrada em dois estudos com gestantes, ambos com o mesmo instrumento, no Paraguai (33,6\%) 5 e no Brasil $(43,1 \%) 6$. O estudo de Ishida et al. 5 mostrou pre- 
Distribuição da amostra de gestantes, de acordo com as variáveis independentes e as prevalências de transtorno mental comum (TMC) em relação a estas variáveis. Pelotas, Rio Grande do Sul, Brasil, 2007 ( $n=1.267$ ).

\begin{tabular}{|c|c|c|c|}
\hline Variáveis & $\mathbf{n}$ & $\%$ & TMC (\%) \\
\hline \multicolumn{4}{|l|}{ Faixa etária (anos) } \\
\hline $12-18$ & 232 & 18,4 & 40,5 \\
\hline $19-34$ & 912 & 72,2 & 41,8 \\
\hline 35 ou + & 120 & 9,5 & 40,8 \\
\hline \multicolumn{4}{|l|}{ Escolaridade } \\
\hline Analfabeta/Fundamental incompleto & 539 & 43,0 & 47,0 \\
\hline Fundamental completo/Médio incompleto & 341 & 27,2 & 43,5 \\
\hline Médio completo/Superior & 373 & 29,8 & 31,6 \\
\hline \multicolumn{4}{|l|}{ Classificação socioeconômica } \\
\hline$A$ e $B$ & 123 & 9,8 & 31,7 \\
\hline C & 674 & 53,6 & 38,1 \\
\hline$D$ e $E$ & 461 & 36,6 & 48,8 \\
\hline \multicolumn{4}{|l|}{ Trabalho } \\
\hline Não & 871 & 69,6 & 43,9 \\
\hline $\operatorname{Sim}$ & 381 & 30,4 & 35,5 \\
\hline \multicolumn{4}{|l|}{ Estado civil } \\
\hline Solteira, divorciada ou viúva & 338 & 26,8 & 44,3 \\
\hline Casada ou vive com o companheiro & 925 & 73,2 & 40,2 \\
\hline \multicolumn{4}{|l|}{ Trimestre gestacional } \\
\hline Segundo trimestre & 793 & 62,6 & 42,4 \\
\hline Terceiro trimestre & 474 & 37,4 & 39,9 \\
\hline \multicolumn{4}{|l|}{ Apoio familiar } \\
\hline Não & 55 & 4,4 & 65,5 \\
\hline $\operatorname{Sim}$ & 1.203 & 95,6 & 40,0 \\
\hline \multicolumn{4}{|l|}{ Pensou em abortar } \\
\hline Não & 1.141 & 90,6 & 38,3 \\
\hline $\operatorname{Sim}$ & 119 & 9,4 & 70,3 \\
\hline \multicolumn{4}{|l|}{ Consumo de tabaco } \\
\hline Não & 1.019 & 80,7 & 38,5 \\
\hline Sim & 244 & 19,3 & 53,1 \\
\hline \multicolumn{4}{|l|}{ Consumo de bebida alcoólica } \\
\hline Não & 1.105 & 91,8 & 40,0 \\
\hline $\operatorname{Sim}$ & 99 & 8,2 & 58,6 \\
\hline Total & 1.267 & 100,0 & 41,4 \\
\hline
\end{tabular}

valência significativamente maior de TMC entre as gestantes $(33,6 \%)$ quando comparadas a mulheres não grávidas (25,8\%). Entretanto, encontrou-se discrepância quanto aos achados de uma pesquisa com gestantes $(20,2 \%) 23$ que utiliza um instrumento considerado padrão ouro na avaliação de transtornos psiquiátricos, The Clinical Interview Schedule - Revised Version (CIS-R), assim, acredita-se que esta discrepância se deve ao diferente instrumento de aferição. Por outro lado, salienta-se que o SRQ-20 é um instrumento breve e auto-aplicável, recomendável para qualquer pro- fissional da área da saúde, uma vez que poucas mulheres têm acesso à avaliação psicológica durante a gestação, enquanto o acompanhamento pré-natal em geral não oferece este serviço. Em Pelotas, a cobertura do serviço público de saúde no atendimento a gestantes é de $51 \% 24$, assim, se a equipe de saúde utilizar um instrumento de screening para avaliar transtornos mentais podese fortalecer as alternativas para a melhoria da qualidade da atenção.

As características que sugerem vulnerabilidade social para a vida da gestante, como o baixo 
Razões de prevalência (RP) não ajustadas e ajustadas das variáveis independentes em relação ao transtorno mental comum (TMC), de acordo com o modelo hierárquico.

\begin{tabular}{|c|c|c|c|c|}
\hline Variáveis & $\begin{array}{c}\text { RP não } \\
\text { ajustadas } \\
\text { (IC95\%) }\end{array}$ & Valor de $p$ & $\begin{array}{l}\text { RP ajustadas } \\
\text { (IC95\%) }\end{array}$ & Valor de $p$ \\
\hline \multicolumn{5}{|l|}{ Primeiro nível hierárquico } \\
\hline Escolaridade & & $<0,001$ & & 0,009 \\
\hline Analfabeta/Fundamental incompleto & $1,48(1,24-1,76)$ & & $1,30(1,07-1,58)$ & \\
\hline Fundamental completo/Médio incompleto & $1,37(1,13-1,66)$ & & $1,28(1,05-1,56)$ & \\
\hline Médio completo/Superior & Referência & & Referência & \\
\hline Classificação socioeconômica & & $<0,001$ & & 0,014 \\
\hline$A \in B$ & Referência & & Referência & \\
\hline C & $1,20(0,91-1,58)$ & & $1,08(0,82-1,42)$ & \\
\hline $\mathrm{D}$ e $\mathrm{E}$ & $1,53(1,16-2,02)$ & & $1,29(0,97-1,71)$ & \\
\hline Trabalho & & 0,007 & & 0,092 \\
\hline Não & $1,23(1,05-1,44)$ & & $1,14(0,96-1,34)$ & \\
\hline Sim & Referência & & Referência & \\
\hline Estado civil & & 0,181 & & 0,463 \\
\hline Solteira, divorciada ou viúva & $1,10(0,95-1,27)$ & & $1,04(0,90-1,21)$ & \\
\hline Casada ou vive com o companheiro & Referência & & Referência & \\
\hline \multicolumn{5}{|l|}{ Segundo nível hierárquico } \\
\hline Apoio familiar & & $<0,001$ & & 0,004 \\
\hline Não & $1,63(1,33-2,00)$ & & $1,34(1,10-1,63)$ & \\
\hline Sim & Referência & & Referência & \\
\hline Pensou em abortar & & $<0,001$ & & $<0,001$ \\
\hline Não & Referência & & Referência & \\
\hline Sim & $1,83(1,59-2,10)$ & & $1,61(1,39-1,87)$ & \\
\hline \multicolumn{5}{|l|}{ Terceiro nível hierárquico } \\
\hline Consumo de tabaco & & $<0,001$ & & 0,301 \\
\hline Não & Referência & & Referência & \\
\hline Sim & $1,37(1,19-1,58)$ & & $1,07(0,93-1,23)$ & \\
\hline Consumo de bebida alcoólica & & $<0,001$ & & 0,164 \\
\hline Não & Referência & & Referência & \\
\hline Sim & $1,46(1,22-1,75)$ & & $1,12(0,95-1,32)$ & \\
\hline Auto-estima & $1,11(1,09-1,12)$ & $<0,001$ & $1,09(1,08-1,11)$ & $<0,001$ \\
\hline
\end{tabular}

nível de escolaridade, menor classificação socioeconômica e pouco apoio familiar estão associadas à maior probabilidade da mulher apresentar TMC durante a gestação. Esse achado, confirmado por outros estudos 5,6,8,11,13,23, implica a hipótese de que, além dos fatores específicos do período gestacional e da auto-estima da gestante, as condições de vida da mulher neste período são importantes preditores de transtornos mentais.

$\mathrm{O}$ fato de ter pensado em aborto demonstrou relação significativa com a presença de TMC, conforme encontramos na pesquisa realizada em Recife ${ }^{6}$. No referido estudo, a prevalência de TMC entre gestantes que pensaram em abortar foi de 51,6\%; estes resultados sugerem que as mulheres grávidas com transtornos mentais estão mais propensas a idealizar o aborto.

A auto-estima da gestante mostrou-se associada com TMC nesta pesquisa, ou seja, as mulheres com menor auto-estima apresentavam maior probabilidade de transtornos mentais. Embora não tenha sido encontrado nenhum estudo específico com essa relação, observa-se que os achados mais próximos a esse resultado correspondem a um estudo realizado no Nordeste com mães adolescentes que provocaram o aborto, apresentando menor auto-estima quando comparadas àquelas que decidiram não in- 
terromper a gravidez 13 . Além desse, uma pesquisa realizada com gestantes em Pelotas mostrou associação entre menor auto-estima e mal-estar psicológico 25. Cabe-se salientar que a média de auto-estima encontrada $(9,3 \pm 4,8)$ é menor que o valor central da escala (15 pontos), entretanto, um estudo sobre a relação entre fadiga e autoestima encontrou média de 7,3 $\pm 3,8$ em pacientes com esclerose múltipla 26. Justificando, assim, que as mudanças que acompanham a gestação podem afetar a auto-estima.

Conclui-se que houve uma significativa associação entre maior prevalência de TMC e menor auto-estima, evidenciando o impacto dos transtornos mentais sobre as competências maternas e a qualidade na interação mãe/bebê 15. Esperase com este estudo contribuir para a melhoria

\section{Resumo}

O objetivo do presente estudo foi verificar a prevalência de transtornos mentais comuns (TMC) e sua relação com auto-estima, bem como outros fatores associados à ocorrência de TMC em gestantes. Foi realizado um estudo transversal aninhado a uma coorte no qual participaram gestantes atendidas no serviço de saúde do Sistema Único de Saúde na cidade de Pelotas, Estado do Rio Grande do Sul, Brasil. Foi utilizado para o rastreamento de transtornos mentais comuns o Self-Reporting Questionnaire (SRQ-20) e, para avalia a auto-estima, $a$ Escala de Auto-Estima de Rosenberg. A amostra constituiu-se de 1.267 gestantes, que tinham em média 25 anos $(d p=6,53)$. A média de auto-estima foi de 9,3 pontos $(d p=4,76)$ e a prevalência de TMC em gestantes foi de 41,4\%. Evidenciou-se, também, que quanto menor a auto-estima da grávida maiores são as chances de associação a TMC $(p<0,001)$. Houve uma significativa associação entre maior prevalência de TMC e baixa auto-estima.

Gravidez; Autoestima; Transtornos Mentais na qualidade da atenção pré-natal nos serviços de saúde. É necessário que se materializem estratégias de prevenção e promoção à saúde mental mãe/bebê, e suas conseqüências na saúde física, com base em um maior conhecimento sobre as variáveis que interferem diretamente no processo.

Sugere-se que sejam realizados estudos longitudinais sobre TMC e auto-estima em gestantes, para que possibilitem um maior entendimento sobre o desenvolvimento destes fatores na gestação e no pós-parto. A gravidez é um período de grande suscetibilidade, e a interação entre TMC e menor auto-estima pode trazer conseqüências negativas para a díade mãe/bebê e para o desenvolvimento infantil.

\section{Colaboradores}

R. A. Silva participou do projeto, orientou a realização de todas as etapas da pesquisa, revisou o artigo e a análise dos dados, e trabalhou na redação final. L. C. Ores participou do projeto, revisão bibliográfica, elaboração do trabalho de campo, análise dos dados, discussão dos resultados e redação do manuscrito. T. C. Mondin participou da revisão bibliográfica, elaboração do trabalho de campo, discussão dos resultados e redação do manuscrito. R. N. Rizzo participou da revisão bibliográfica, elaboração do trabalho de campo, discussão dos resultados e redação do manuscrito. I. G. S. Moraes participou da concepção do projeto e discussão dos resultados, bem como aprovou a versão final a ser publicada. K. Jansen participou da concepção do projeto, elaboração do trabalho de campo e discussão dos resultados, bem como aprovou a versão final a ser publicada. R. T. Pinheiro participou do projeto, orientou a realização de todas as etapas da pesquisa e aprovou a versão final a ser publicada.

\section{Agradecimentos}

À Coordenação de Aperfeiçoamento de Pessoal de Nível Superior (CAPES) pelo apoio financeiro. A todos os colegas que possibilitaram a realização da pesquisa e/ ou pessoas que colaboraram de alguma forma com o estudo. 


\section{Referências}

1. Falcone VM, Mäder CVN, Nascimento CFL, Santos JMM, Nóbrega FJ. Multiprofessional care and mental health in pregnant women. Rev Saúde Pública 2005; 39:612-8.

2. Baptista MN, Baptista ASD, Torres ECR. Associação entre suporte social, depressão e ansiedade em gestantes. Psic Rev Psicol Vetor Ed 2006; 7:39-48.

3. Goldberg D, Huxley P. Common mental disorders: a bio-social model. London: Tavistock; 1992.

4. Pinheiro KAT, Horta BL, Pinheiro RT, Horta LL, Terres NG, Silva RA. Common mental disorders in adolescents: a population based cross-sectional study. Rev Bras Psiquiatr 2007; 29:241-5.

5. Ishida K, Stupp P, Serbanescu F, Tullo E. Perinatal risk for common mental disorders and suicidal ideation among women in Paraguay. Int J Gynaecol Obstet 2010; 110:235-40.

6. Ludermir AB, Araújo TV, Valongueiro SA, Lewis G. Common mental disorders in late pregnancy in women who wanted or attempted an abortion. Psychol Med 2009; 26:1-7.

7. PatelV, Kirkwood BR, Pednekar S, Pereira B, Barros P, Fernandes J, et al. Gender disadvantage and reproductive health risk factors for common mental disorder in women: a community survey in India. Arch Gen Psychiatry 2006; 63:404-13.

8. Maragno L, Goldbaum M, Gianini RJ, Novaes HMD, César CLG. Prevalência de transtornos mentais comuns em populações atendidas pelo Programa Saúde da Família (QUALIS) no Município de São Paulo, Brasil. Cad Saúde Pública 2006; 22:1639-48.

9. Veggi AB, Lopes CS, Faerstein E, Sichieri R. Índice de massa corporal, percepção do peso corporal e transtornos mentais comuns entre funcionários de uma universidade no Rio de Janeiro. Rev Bras Psiquiatr 2004; 26:242-7.

10. Piccinelli M, Wilkinson G. Gender differences in depression: critical review. Br J Psychiatry 2000; 177:486-92.

11. Evans DL, Charney DS, Lewis L, Golden RN, Gorman JM, Krishnan KR, et al. Mood disorders in the medically ill: scientific review and recommendations. Biol Psychiatry 2005; 58:175-89.

12. Gonçalves DM, Kapczinski F. Transtornos mentais em comunidade atendida pelo Programa Saúde da Família. Cad Saúde Pública 2008; 24:1641-50.

13. Hanlon C, Medhin G, Alem A, Tesfaye F, Lakew Z, Worku B, et al. Impact of antenatal common mental disorders upon perinatal outcomes in Ethiopia: the P-MaMiE population-based cohort study. Trop Med Int Health 2009; 14:156-66.

14. Gobitta M, Guzzo RSL. Estudo inicial do inventário de auto-estima (SEI) - Forma A. Psicol Reflex Crít 2002; 15:143-50.
15. Dubow EF, Luster T. Adjustment of children born to teenage mothers: the contribution of risk and protective factors. J Marriage Fam 1990; 52:393-404.

16. Robinson JP, Shaver PR, Wrightman LS. Measures of personality and social psychological attitudes. New York: Academic Press; 1991.

17. Gonçalves DM, Stein AT, Kapczinski F. Avaliação de desempenho do Self-Reporting Questionnaire como instrumento de rastreamento psiquiátrico: um estudo comparativo com o Structured Clinical Interview for DSM-IV-TR. Cad Saúde Pública 2008; 24:380-90.

18. Dini GM. Tradução para a língua portuguesa, adaptação cultural e validação do questionário de auto-estima de Rosenberg [Dissertação de Mestrado]. São Paulo: Escola Paulista de Medicina, Universidade Federal de São Paulo; 2004.

19. Masur J, Monteiro MG. Validation of the CAGE alcoholism screening test in a Brazilian psychiatric inpatient hospital setting. Braz J Med Biol Res 1983; 16:215-8.

20. Victora CG, Huttly SR, Fuchs SC, Olinto MT. The role of conceptual frameworks in epidemiological analysis: a hierarchical approach. Int J Epidemiol 1997; 26:224-7.

21. Lima MCP, Menezes PR, Carandina L, Cesar CLG, Barros MBA, Goldbaum M. Transtornos mentais comuns e uso de psicofármacos: impacto das condições socioeconômicas. Rev Saúde Pública 2008; 42:717-23.

22. Marín-León L, Oliveira HB, Barros MBA, Dalgalarrondo P, Botega NJ. Social inequality and common mental disorders. Rev Bras Psiquiatr 2007; 29:250-3.

23. Faisal-Cury A, Menezes P, Araya R, Zugaib M. Common mental disorders during pregnancy: prevalence and associated factors among low-income women in São Paulo, Brazil: depression and anxiety during pregnancy. Arch Womens Ment Health 2009; 12:335-43.

24. Araújo TM, Pinho PS, Almeida MMG. Prevalence of psychological disorders among women according to socio demographic and housework characteristics. Rev Bras Saúde Mater Infant 2005; 5:337-48.

25. Dias MS, Silva RA, Souza LDM, Lima RC, Pinheiro RT, Moraes IGS. Self-esteem and associated factors in pregnant women in the city of Pelotas, Rio Grande do Sul State, Brazil. Cad Saúde Pública 2008; 24:2787-97.

26. Fragoso YD, Silva EO, Finkelsztejn A. Correlation between fatigue and self-esteem in patients with multiple sclerosis. Arq Neuropsiquiatr 2009; 67:818-21.

Recebido em 07/Abr/2010

Versão final reapresentada em 16/Jul/2010 Aprovado em 26/Jul/2010 